\title{
CROSS-CORRELATION-BASED METHOD VS. CLASSICAL FFT FOR SPECTRAL ANALYSIS OF IMPULSE RESPONSE
}

\author{
Adam KOTOWSKI ${ }^{*}$ \\ ${ }^{*}$ Faculty of Mechanical Engineering, Department of Automatic Control and Robotics, Bialystok University of Technology, \\ ul. Wiejska 45C, 15-351 Bialystok, Poland

\section{a.kotowski@pb.edu.pl}

\begin{abstract}
The paper presents comparison of results of impulse response spectral analysis that has been obtained using a method based on cross-correlation with results obtained using classical FFT. The presented non-Fourier method is achieved by correlating the analyzed signal and reference single-harmonic signals and using Hilbert transform to obtain an envelope of cross-correlation. The envelope of crosscorrelation makes it possible to calculate appropriate indicator and make its plot in frequency domain as a spectrum. The spectrum obtained this way has its advantage over the FFT that the spectral resolution does not depend on duration of signal. At the same time, the spectral resolution can be much greater than spectral resolution resultant from FFT. Obtained results show that presented non-Fourier method gives frequency readout more accurate in comparison to FFT when the impulse response is a short-time signal e.g. few dozen of miliseconds lasting.
\end{abstract}

Key words: Spectral Analysis, FFT, Cross-Correlation, Hilbert transform, Envelope, Spectral Resolution

\section{INTRODUCTION}

Impulse response as a result of impact testing has been used in wide area of engineering owing to its convenience and simplicity on experimentation (Ahn et al., 2005). It is well known that using fast Fourier transform (FFT) for spectrum analysis will give an immediate frequency profile of recorded signals. When analyzing signals using FFT, frequency resolution is fixed as an inverse of the duration of the analyzed signal (Bendat and Piersol, 1980; Gasior, 2004) and, as a result, it fails to meet the requirements of measurement. One of the ways to increase frequency resolution and improve frequency reading is interpolation. It improves the resolution by a few orders, depending on the interpolation method (Gasior, 2006; Gasior, 2010). In order to avoid limitation in frequency resolution using FFT, it has been investigated the problem of frequency resolution and showed that it was possible to obtain frequency resolution of one-tenth of the spacing between the frequency points produced by the Fourier transform (Cawley and Adams, 1979). For increasing spectral frequency resolution and improving frequency estimation, the zero-padding technique also is widely used (Quinn, 2009; Dunne, 2002). In general, the interpolation algorithms can be a computing-cost-effective replacement of the zero-padding technique in applications (Gasior, 2006).

There are a great number of engineering applications of correlation function (Bendat and Piersol, 1980). Looking for its new applications, the cross-correlation function has been utilized to correlate real-measured signal and a single-harmonic signal generated by software (Kotowski, 2010). Also, the Hilbert transform was used to obtain the envelope of the cross-correlation function (Thrane, 1984; Thrane et al., 1999).

This paper describes a method of reading a particular frequency of the harmonic developed on the basis of the cross-correlation function and its envelope. The main advantage of this method over the FFT is its ability to obtain different frequency resolution from that obtained by using FFT, often required as increased resolution, e.g. ten times increased.

\section{METHODOLOGY}

The cross-correlation function $R_{x y}(\tau)$ between two processes, $x(t)$ and $y(t)$, is calculating by the expression (Bendat and Piersol, 1980):

$R_{x y}(\tau)=\lim _{T \rightarrow \infty} \frac{1}{T} \int_{0}^{T} x(t) y(t+\tau) d t$

where: $T$ - signal record length, $\tau$ - argument of cross-correlation function (time delay).

The Hilbert Transform (HT) enables calculation of the envelope $A(t)$ of a real-valued function $x(t)$ as follows (Thrane et al., 1999; Feldman, 2011):

$A(t)=\sqrt{x^{2}(t)+\tilde{x}^{2}(t)}$

where $\tilde{x}(t)$ is HT of a real-valued function $x(t)$. Then, an envelope of the cross-correlation function Renv is as follows:

$R_{e n v}(\tau)=\sqrt{R_{x y}{ }^{2}(\tau)+\tilde{R}_{x y}{ }^{2}(\tau)}$

where: $\tilde{R}_{x y}$ is HT of the cross-correlation $R_{x y}$. The envelope of the cross-correlation of two harmonic processes with one of them being damped, decays exponentially. In addition, the equality of the frequencies of the harmonics creates the highest position of the envelope plot in comparison to the non-equality of the frequencies (Kotowski, 2014). Apart from analyzed signal $y(t)$, the method used in the paper requires a series of harmonic signals gi $(t)$ generated as follows:

$g_{i}=\sin (2 \pi \cdot w \cdot i \cdot t)$

where: $i$ - an integer value (index), $w$ - frequency resolution. 
After correlating the input and the generated signals, the plot position of the envelope of the cross-correlation indicates an identification of the harmonic detected. This phenomenon is easy to observe and the determination of common frequency is simple. As a result, the envelope plot can be effectively used to identify the harmonics incorporated in recorded signals with no need for the Fourier transform. The frequency value of the harmonic in the input signal $y(t)$ can be determined using the envelope plot. Studying the envelopes, we obtain both $w$ and $i$ values of signal $g_{i}(t)$ used for calculations. Thus, formula $(w \cdot i)$ indicates the frequency of the identified harmonic. Apart from cross-correlation envelope plots, indicator $S_{e}$ is used to express envelope position numerically as follows:

$S_{e}(n)=\Delta t \sum_{n=1}^{N} R_{e n v}(n)$

where $R_{\text {env }}$ is the cross-correlation envelope, $\Delta t$ is the sampling period and $N$ stands for the number of samples. As a result, it is possible to prepare the $S_{e}$ plot, as a result of spectral analysis, for a frequency span in which the impulse response has been recorded. The frequency resolution of $S_{e}$ plot is strictly connected to $w$ value.

\section{DATA FOR ANALYSIS}

To illustrate the comparison between cross-correlation-based method and FFT for spectral analysis of impulse response, a numerical example of a four-degree-of-freedom system impulse response is considered.

The unit impulse response function of a multi-degree-of-freedom system can be expressed as (Ahn et al., 2005):

$h(t)=\sum_{r=1}^{n} A_{r} \exp \left(-\sigma_{r} t\right) \sin \left(\omega_{d r} t\right)$

where: $A_{r}$ - the $r$ th modal constant, $\sigma_{r}$ - the $r$ th modal damping, $\omega_{d r}$ the $r$ th damped angular frequency of the system. Thus, fourdegree-of-freedom system impulse response can be expressed as:

$$
\begin{aligned}
h(t)= & A_{1} \exp \left(-\sigma_{1} t\right) \sin \left(2 \pi f_{1} t\right) \\
& +A_{2} \exp \left(-\sigma_{2} t\right) \sin \left(2 \pi f_{2} t\right) \\
& +\ldots+A_{3} \exp \left(-\sigma_{3} t\right) \sin \left(2 \pi f_{3} t\right) \\
& \quad+A_{4} \exp \left(-\sigma_{4} t\right) \sin \left(2 \pi f_{4} t\right)
\end{aligned}
$$

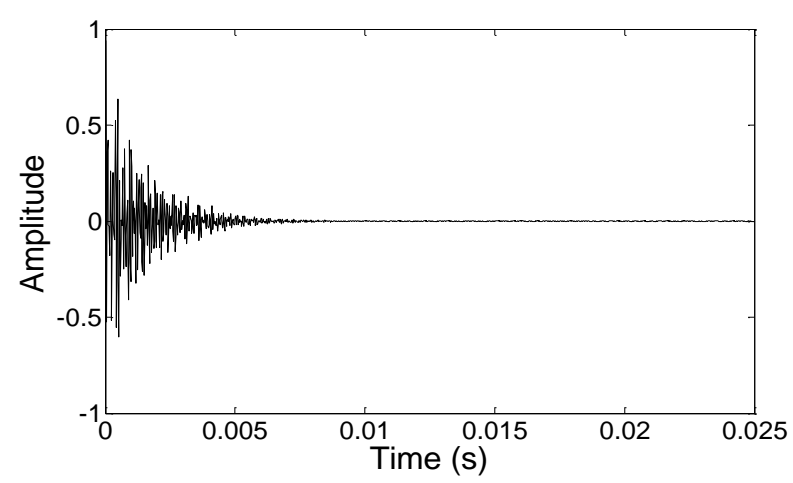

Fig. 1. Impulse response for analysis

For obtaining time history of four-degree-of-freedom system impulse response, values of parameters of Eq. (7) have been assumed. They are: $A_{1}=0.13, A_{2}=0.40, A_{3}=0.23$, $A_{4}=0.30, \sigma_{1}=560, \sigma_{2}=610, \sigma_{3}=640, \sigma_{4}=600$, $f_{1}=3196.4 \mathrm{~Hz}, \quad f_{2}=11027.3 \mathrm{~Hz}, \quad f_{3}=13244.5 \mathrm{~Hz}$, $f_{4}=15168.7 \mathrm{~Hz}$. The considered impulse response have been calculated by sampling frequency equal to $48000 \mathrm{~Hz}$ and sampling time $T$ equal to $0.025 \mathrm{~s}$. The impulse response under consideration is shown in Fig.1. In fact, the impulse response goes to zero after the time of $0.010 \mathrm{~s}$. These two periods of time history are to be considered in the next Section.

\section{RESULTS}

In this section, it is presented results of impulse response spectral analysis by using cross-correlation-based (CCB) method and FFT. These kinds of analysis are compared on the basis of values of frequencies readout for four spectra peaks.

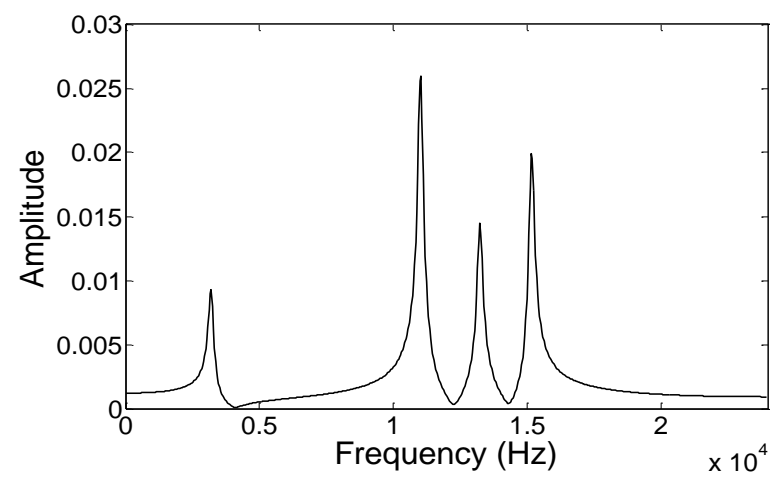

Fig. 2. Impulse response spectrum obtained using FFT for $T=0.025 \mathrm{~s}$

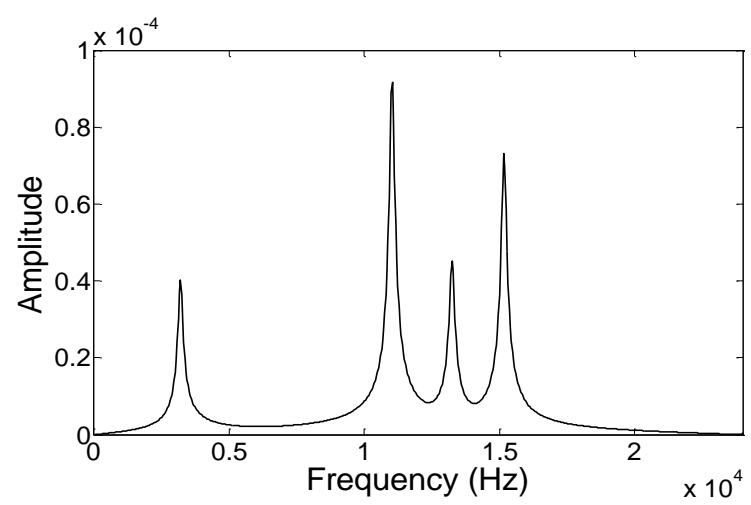

Fig. 3. $S_{e}$ indicator plot for $T=0.025 \mathrm{~s}$ and $\Delta f=40 \mathrm{~Hz}$

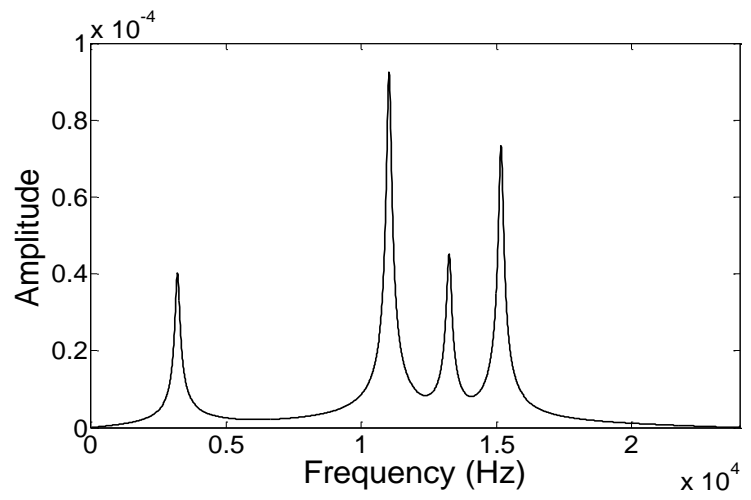

Fig. 4. $S_{e}$ indicator plot for $T=0.025 \mathrm{~s}$ and $\Delta f=10 \mathrm{~Hz}$ 


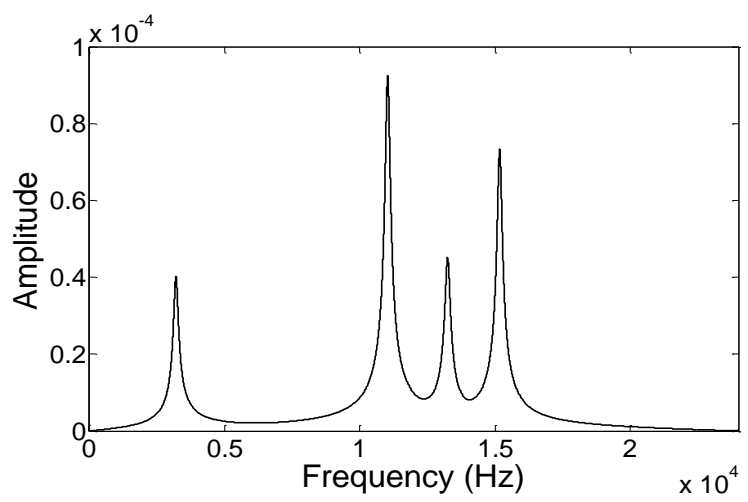

Fig. 5. $S_{e}$ indicator plot for $T=0.025 \mathrm{~s}$ and $\Delta f=1 \mathrm{~Hz}$

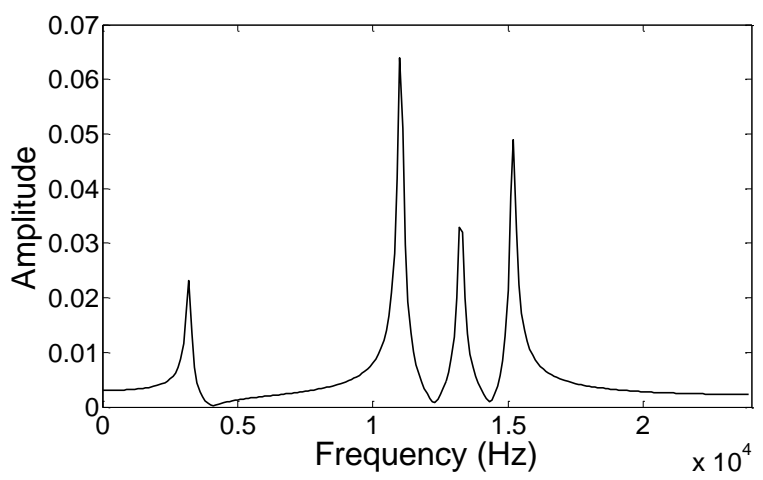

Fig. 6. Impulse response spectrum obtained using FFT for $T=0.010 \mathrm{~s}$

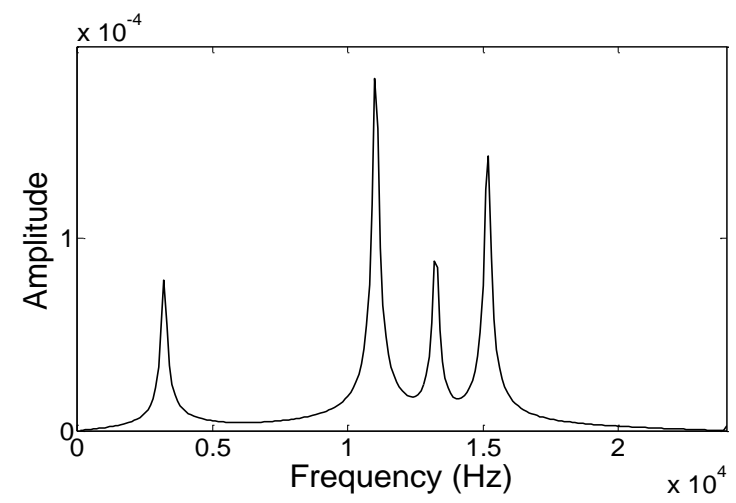

Fig. 7. $S_{e}$ indicator plot for $T=0.010 \mathrm{~s}$ and $\Delta f=100 \mathrm{~Hz}$

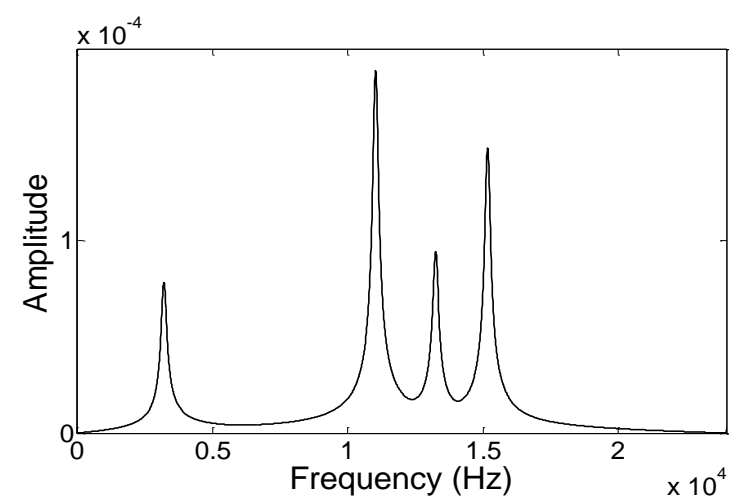

Fig. 8. $S_{e}$ indicator plot for $T=0.010 \mathrm{~s}$ and $\Delta f=10 \mathrm{~Hz}$

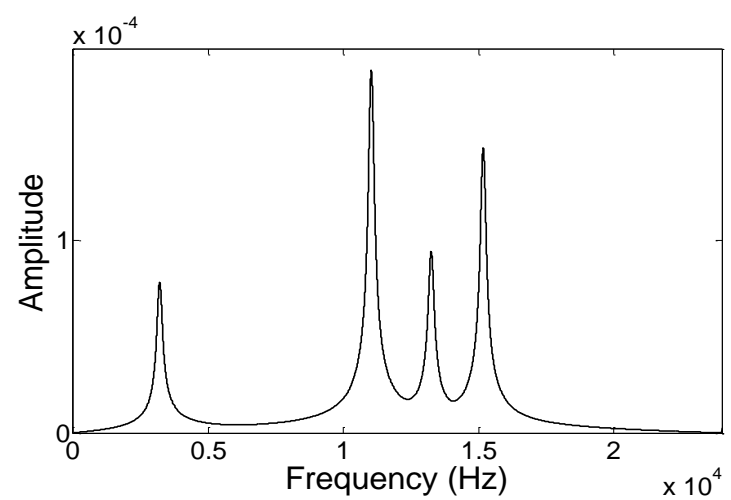

Fig. 9. $S_{e}$ indicator plot for $T=0.010 \mathrm{~s}$ and $\Delta f=1 \mathrm{~Hz}$

The spectral analysis using FFT has resulted frequencies readout by frequency resolution $\Delta f$ as a result of the sampling time of impulse response $T$. It has been $40 \mathrm{~Hz}$ and $100 \mathrm{~Hz}$ for 0.025 second and 0.010 second, respectively. The spectral analysis using CCB method has resulted frequencies readout by different frequency resolution independent of the sampling time of impulse response.

The spectra obtained using FFT and the Se indicator (Eq.5) for $T=0.025$ second and $T=0.010$ second are shown in Figs 2-5 and Figs. 6-9, respectively.

The values of frequencies read from plots for both methods are shown in Tabs. 1 and 2 for sampling tine equal to $0.025 \mathrm{~s}$ and $0.010 \mathrm{~s}$, respectively. There are four frequency values for succeeding four plot peaks. The one of CCB method frequency resolution has been fixed the same as for FFT $(40 \mathrm{~Hz}, 100 \mathrm{~Hz})$. For further research of CCB method, resolution of $10 \mathrm{~Hz}$ and $1 \mathrm{~Hz}$ has also been considered for that method.

Tab. 1. Results for sampling time $T=0.025 \mathrm{~s}$

\begin{tabular}{|c|c|c|c|c|}
\hline $\begin{array}{c}\text { Theoretical } \\
\text { frequency } \\
(\mathbf{H z})\end{array}$ & $\begin{array}{c}\mathrm{FFT} \\
\Delta \mathrm{f}=\mathbf{4 0 H z}\end{array}$ & $\begin{array}{c}\text { CCB } \\
\text { method } \\
\Delta \mathbf{f}=\mathbf{4 0 H z}\end{array}$ & $\begin{array}{c}\text { CCB } \\
\text { method } \\
\Delta \mathbf{f = 1 0 H z}\end{array}$ & $\begin{array}{c}\text { CCB } \\
\text { method } \\
\Delta \mathbf{f = 1 H z}\end{array}$ \\
\hline 3196.4 & 3200 & 3200 & 3200 & 3198 \\
\hline 11027.3 & 11040 & 11040 & 11030 & 11028 \\
\hline 13244.5 & 13240 & 13240 & 13240 & 13244 \\
\hline 15168.7 & 15160 & 15160 & 15170 & 15169 \\
\hline
\end{tabular}

Tab. 2. Results for sampling time $T=0.010 \mathrm{~s}$

\begin{tabular}{|c|c|c|c|c|}
\hline $\begin{array}{c}\text { Theoretical } \\
\text { frequency } \\
\text { (Hz) }\end{array}$ & $\begin{array}{c}\mathrm{FFT} \\
\Delta \mathrm{f}=\mathbf{1 0 0 H z}\end{array}$ & $\begin{array}{c}\mathrm{CCB} \\
\text { method } \\
\mathbf{\Delta f = 1 0 0 H z}\end{array}$ & $\begin{array}{c}\mathbf{C C B} \\
\text { method } \\
\Delta \mathrm{f}=10 \mathrm{~Hz}\end{array}$ & $\begin{array}{c}\mathbf{C C B} \\
\text { method } \\
\Delta \mathrm{f}=1 \mathrm{~Hz}\end{array}$ \\
\hline 3196.4 & 3200 & 3200 & 3200 & 3199 \\
\hline 11027.3 & 11000 & 11000 & 11030 & 11028 \\
\hline 13244.5 & 13200 & 13200 & 13240 & 13244 \\
\hline 15168.7 & 15200 & 15200 & 15170 & 15169 \\
\hline
\end{tabular}

The main difference between methods is connected with the errors between determined frequencies and theoretical frequencies. The CCB method allows to obtain higher frequency resolution than classical FFT. It resulted less errors between determined frequencies and theoretical frequencies. Hence, frequency determination has been improved in comparison to classical FFT. Tabs. 1 and 2 also show that results obtained by CCB method do not depend on sampling time, they are the same or difference seems very 
negligible. For the frequency resolution of $1 \mathrm{~Hz}$, the CCB method resulted the lowest error for both signal lengths. In this case, absolute errors between theoretical frequency and measured frequency reached $2.6(1.6) \mathrm{Hz}, 0.7 \mathrm{~Hz}, 0.5 \mathrm{~Hz}$ and $0.3 \mathrm{~Hz}$. Using $\mathrm{FFT}$, this errors reached varies from 3.6 to $44.5 \mathrm{~Hz}$. Figs. 10 and 11 show relation in frequency determination errors for two lengths of analyzed impulse response, $0.025 \mathrm{~s}$ and $0.010 \mathrm{~s}$, respectively.

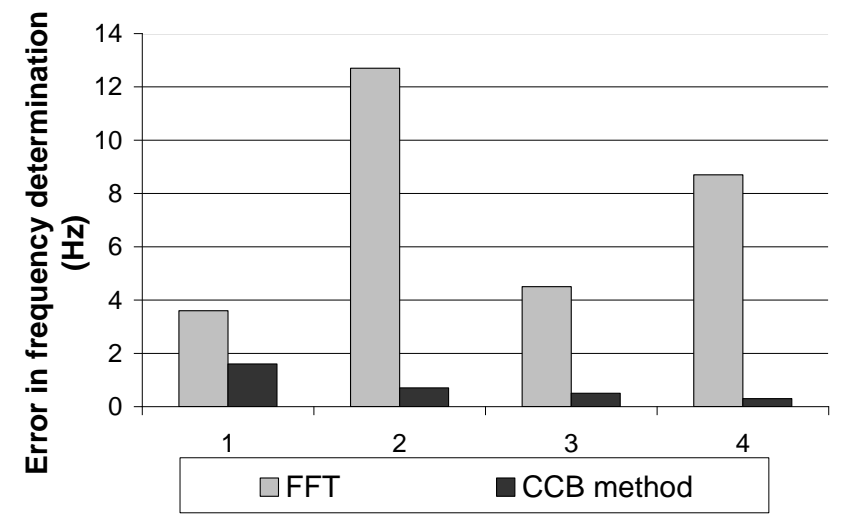

Fig. 10. Relation in frequency determination errors for FFT and CCB method for sampling time $T=0.025 \mathrm{~s}$

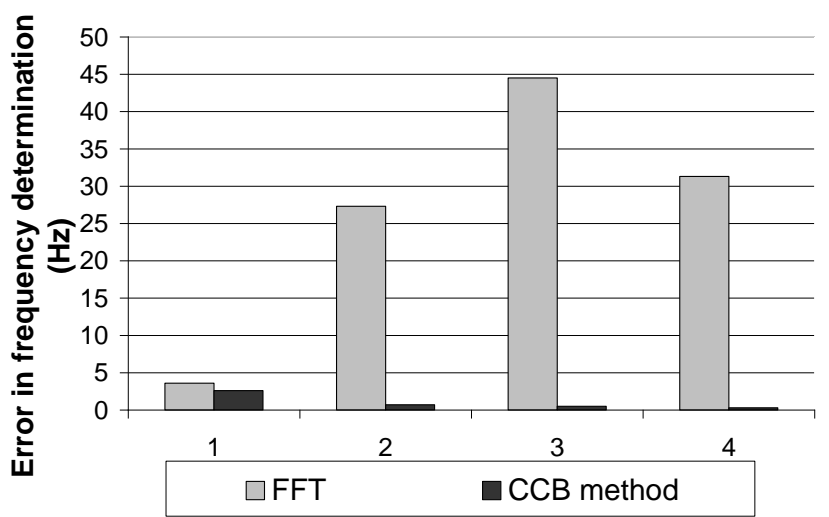

Fig. 11. Relation in frequency determination errors for FFT and CCB method for sampling time $T=0.010 \mathrm{~s}$

Except peak near theoretical frequency of $3196.4 \mathrm{~Hz}$, the frequency errors using CCB method $(0.3 \div 0.7 \mathrm{~Hz})$ are several dozen times lower than frequencies errors using FFT $(4.5 \div 44.5 \mathrm{~Hz})$.

\section{CONCLUSIONS}

The paper presents comparison of results of frequency reading based on impulse response spectral analysis that has been obtained using cross-correlation-based method with results obtained using classical FFT. The presented non-Fourier method is achieved by correlating the analyzed signal and reference single-harmonic signals and using Hilbert transform to obtain an envelope of crosscorrelation. The spectral analysis obtained using proposed method has its advantage over the FFT that the spectral resolution does not depend on signal length and it gives a possibility to obtain a controllable spectral resolution. Moreover, the spectral resolution can be much greater than spectral resolution resultant from FFT. By extension, frequency readings are more accurate by doing proposed frequency resolution improvement. Thus, cross-correlation-based method can be an additional method for improving the accuracy of natural frequencies measurement using impulse tests.

\section{REFERENCES}

1. Ahn S. J., Jeong W. B., Yoo W. S. (2005), Improvement of impulse response spectrum and its application, Journal of Sound and Vibration, 288, 1223-1239.

2. Bendat J. S., Piersol A. G. (1980), Engineering applications of correlation and spectral analysis, Wiley-Interscience, New York.

3. Cawley P., Adams R. D. (1979), Improved frequency resolution from transient tests with short record lengths, Journal of Sound and Vibration, Vol. 64, 123-132.

4. Dunne J. F. (2002), A fast time-domain integration method for computing non-stationary response histories of linear oscillators with discrete-time random forcing, Journal of Sound and Vibration, Vol. 254, 635-676.

5. Feldman M. (2011), Hilbert transform in vibration analysis, Mechanical Systems and Signal Processing, 25, 735-802.

6. Gasior M. (2006), Improving frequency resolution of discrete spectra, Ph.D. thesis, AGH University of Science and Technology, Krakow, Poland.

7. Gasior M. (2010), Improving frequency resolution of discrete spectra - Algorithms of three-node interpolation, ISBN 978-3-8383-5943-4, LAP LAMBERT Academic Publishing.

8. Gasior M., Gonzalez J. L. (2004), Improving FFT frequency measurement resolution by parabolic and gaussian interpolation, AB-Note-2004-021 BDI, CERN, Geneva, Switzerland.

9. Kotowski A. (2010), Reading the frequency of harmonics by crosscorrelation function and its envelope, Proc. 6th Int. Conf. Mechatronic Systems and Materials, 108-109.

10. Kotowski A. (2014), A new method for spectral analysis of nonstationary signals from impact tests, Journal of Vibroengineering, 16, 2171-2177.

11. Quinn B. G. (2009), Recent advances in rapid frequency estimation, Digital Signal Processing, 19, 942-948.

12. Thrane N. (1984), The Hilbert Transform, Technical Review, No. 3 , Brüel\&Kjær, BV 0015.

13. Thrane N., Wismer J., Konstantin-Hansen H., Gade S. (1999), Practical use of the "Hilbert transform", Application Note, Brüel\&Kjær, BO 0437.

This work was supported by Bialystok University of Technology under work No. S/WM/1/2012. 